\title{
Parotid Incidentaloma Identified by Positron Emission/Computed Tomography: When to Consider Diagnoses Other than Warthin Tumor
}

\author{
Carolina Bothe $^{1}$ Alejandro Fernandez ${ }^{2}$ Jacinto Garcia ${ }^{1}$ Montserrat Lopez ${ }^{1}$ Xavier León ${ }^{1}$ \\ Miquel Quer ${ }^{1}$ Joan Lop ${ }^{1}$
}

\footnotetext{
${ }^{1}$ Department of Otorhinolaryngology, Hospital Sant Pau, Barcelona, Spain

${ }^{2}$ Department of Nuclear Medicine, Hospital de Sant Pau, Barcelona, Barcelona, Spain
}

Address for correspondence Carolina Bothe, MD, Department of Otorhinolaryngology, Hospital Sant Pau, Avda. Sant Antoni Ma Claret, 167, Barcelona 08025, Spain (e-mail: carolina_bothe@hotmail.com).

Int Arch Otorhinolaryngol 2015;19:112-115.

\begin{abstract}
Introduction Parotid gland incidentalomas (PGIs) are unexpected hypermetabolic foci in the parotid region that can be found when scanning with whole-body positron emission/computed tomography (PET/CT). These deposits are most commonly due to benign lesions such as Warthin tumor.

Objective The aim of this study was to determine the prevalence of PGIs identified in $\mathrm{PET} / \mathrm{CT}$ scans and to assess the role of smoking in their etiology.

Methods We retrospectively reviewed all PET/CT scans performed at our center in search of PGIs and identified smoking status and standardized uptake value (SUVmax) in each case. We also analyzed the database of parotidectomies performed in our department in the previous 10 years and focused on the pathologic diagnosis and the presence or absence of smoking in each case.

Results Sixteen cases of PGIs were found in 4,250 PET/CT scans, accounting for $0.4 \%$. The average SUVmax was 6.5 (range 2.8 to 16). Cytology was performed in five patients; it was benign in four cases and inconclusive in one case. Thirteen patients had a history of smoking. Of the parotidectomies performed in our center with a diagnosis of Warthin

Keywords

- parotid gland

- adenolymphoma

- PET scan

- cigarette smoking tumor, we identified a history of smoking in $93.8 \%$ of those patients.

Conclusions The prevalence of PGIs on PET/CT was similar to that reported by other authors. Warthin tumor is frequently diagnosed among PGIs on PET/CT, and it has a strong relationship with smoking. We suggest that a diagnosis other than Warthin tumor should be considered for PGls in nonsmokers.
\end{abstract}

\section{Introduction}

It is not unusual to find unexpected hypermetabolic foci as a result of scanning using whole-body positron emission/computed tomography (PET/CT). Known as incidentalomas, such findings are not necessarily related to the tumor or disease being studied. Parotid gland incidentalomas (PGIs) are defined

received

August 22, 2014

accepted

November 14, 2014

published online

December 29, 2014 as new focal intraglandular deposits of radiotracer in patients without prior history of parotid disease. ${ }^{1}$ These deposits are most commonly due to benign lesions such as Warthin tumor (WT), ${ }^{1-5}$ but they can also be caused by metastatic lymph nodes or malignant tumors. ${ }^{6}$

The parotid gland is the salivary gland that most commonly develops tumors, most of which are benign. After pleomorphic

Copyright $(2015$ by Thieme Publicações License terms Ltda, Rio de Janeiro, Brazil 
adenoma, WT is the second most prevalent parotid tumor. It presents as a slow-growing mass in the tail of the gland, and is usually asymptomatic. It generally occurs between the sixth and seventh decades of life, predominantly in men. It has a strong association with smoking, which is therefore considered a risk factor for its development. ${ }^{7-10}$ WT can be multicentric or bilateral, and it rarely becomes malignant.

To our knowledge, only two previous studies have reported the prevalence of PGI, and both were performed in Asia. ${ }^{2,11}$ Furthermore, clinical features such as the role of smoking have not been considered in their etiology. The present study aimed to determine the prevalence of parotid incidentalomas in ${ }^{18} \mathrm{~F}$-fluorodeoxyglucose $\left({ }^{18} \mathrm{~F}\right.$-FDG $)$ PET/CT and evaluate the presence of smoking in this group of patients. We also reviewed the database of parotidectomies performed in our hospital to assess the relationship between WT and smoking.

\section{Materials and Methods}

\section{Patients}

We conducted a retrospective review of the reports of PET/CT performed in 4,250 patients in our hospital between June 2009 and February 2013. We identified 16 cases of parotid focal uptake in patients without known disease of the parotid glands. The group consisted of 10 men and 6 women, 48 to 88 years of age. In most cases, PET-CT was indicated as an extension study of diagnosed neoplasms or to confirm locoregional relapse after treatment. In two cases, it was indicated to rule out giant cell arteritis.

We reviewed the history of smoking in the 16 cases: 13 had smoked or were current smokers. We identified additional studies performed on the parotid masses, finding that only 5 patients underwent cytologic analysis by fine needle aspiration cytology (FNAC). In 8 of the 16 cases, the lesion was located in the right parotid gland, in 7 cases it was located on the left, and in 1 case it was bilateral. Two patients had more than one deposit within the same gland.

We analyzed the database of parotidectomies performed in our department in the previous 10 years and focused on the pathologic diagnosis and the presence or absence of smoking in each case.

\section{${ }^{18}$ F-Fluorodeoxyglucose Positron Emission/Computed Tomography}

Combined ${ }^{18} \mathrm{~F}$-FDG whole-body PET/CT (Gemini TF, Philips, Amesterdam) with 64-slice CT was performed. Standard patient preparation included: 6-hour fasting, hydration, and serum glucose level of less than $150 \mathrm{mg} / \mathrm{dL}$ before tracer injection. Patients were asked to rest quietly in supine position, and vesical evacuation was done before the acquisition of images.

A dose of $3.7 \mathrm{MBq} / \mathrm{kg}$ of ${ }^{18} \mathrm{~F}$-FDG was intravenously injected. Low-dose CT and PET from the base of the skull to the proximal thighs were performed, with an additional acquisition of head and neck images. The total examination time was $\sim 20$ minutes. An abnormal PET-CT finding was defined as a significant increased uptake, higher than that of the surrounding normal tissue.

\section{Results}

We found PGIs in 16 patients who underwent PET/CT between June 2009 and February 2013, corresponding to $0.4 \%$ of patients undergoing PET/CT. The incidentalomas were more frequent in men $(62.5 \%, 10$ cases $)$, and the average age at diagnosis was 68 years.

The most common indication for the test was lung cancer ( 7 cases, corresponding to $43.75 \%$ ), followed by breast cancer ( 3 cases), giant cell vasculitis ( 1 case each of Horton disease and Takayasu arteritis), and 1 case each of locally advanced oropharyngeal cancer, gallbladder carcinoma with liver metastasis, peritoneal carcinomatosis of gynecologic origin, and retroperitoneal lymphoma. (See - Table 1.)

Five of the 16 patients underwent FNAC of the parotid mass. Three of five masses had an inflammatory component without a malignant component, one was considered a mixed tumor suggestive of pleomorphic adenoma, and the other was inconclusive. Of the 16 patients, only the patient with the pleomorphic adenoma underwent surgery; a superficial parotidectomy was performed and the histology was confirmed. In the remaining 11 cases, FNAC was not performed but the mass was monitored by periodic clinical follow-up. Thirteen patients $(81.25 \%)$ had a history of smoking. One of the three nonsmokers was the patient with the pleomorphic adenoma.

The average standardized uptake value of parotid tumors was 6.51 , ranging from 2.87 to 16.1 . The term used to describe the lesions was hyperintense or hypermetabolic intraglandular nodule.

A review of the database of parotidectomies performed in our department revealed that 311 patients were surgically treated for parotid gland tumors in the previous 10 years and 80 cases $(25.7 \%)$ were identified as WT. This was the second most frequent tumor after pleomorphic adenoma. A history of smoking was identified in $93.8 \%$ of patients with WT (75 cases). This percentage was high compared with the prevalence of smoking in pleomorphic adenomas, which was $38.8 \%$. (See - Table 2.)

\section{Discussion}

Our findings show that the prevalence of PGIs on PET/CT in our institution is similar to that reported in the two previous studies in Asia. When analyzing the presence of cigarette smoking in our patients with PGIs, we found it was a common factor in $81.25 \%$ of the patients.

In our study, FNAC was performed in five cases. Three were negative for malignancy without typing the lesion. One case was a pleomorphic adenoma and the other had a nondiagnostic smear. The remaining patients did not undergo additional testing on the PGI due to the advanced stage of the primary tumor, the absence of symptoms, or the unlikelihood of metastasis based on clinical and tomographic features.

In the largest series published to date, Wang et al found 58 PGIs in 19,333 patients, representing a prevalence of $0.3 \%{ }^{11}$ They obtained histologic confirmation in 51 patients, 41 with benign lesions (mainly represented by pleomorphic adenomas and WT) and 10 with malignant tumors. In a smaller 
114 Parotid Incidentaloma Identified by PET/CT Bothe et al.

Table1 Data of 16 patients with parotid incidentalomas detected by PET/CT

\begin{tabular}{|c|c|c|c|c|c|c|}
\hline Patient & Age (y) & Sex & Smoking & SUVmax & Cytology (FNAC)* & PET/CT indication \\
\hline 1 & 64 & Male & Yes & 4.3 and 3 & Negative for malignancy & Lung cancer \\
\hline 2 & 48 & Female & Yes & 16.1 & Negative for malignancy & Horton disease \\
\hline 3 & 64 & Female & No & 5.1 & Not performed & Breast cancer \\
\hline 4 & 66 & Female & No & 4.55 & Not performed & Breast cancer \\
\hline 5 & 71 & Male & Yes & 4.03 & Inconclusive cytology & Gallbladder cancer \\
\hline 6 & 88 & Female & Yes & 3.3 & Negative for malignancy & Peritoneal carcinomatosis \\
\hline 7 & 54 & Male & Yes & 5.5 & Not performed & Lung cancer \\
\hline 8 & 74 & Male & Yes & 2.87 & Not performed & Lung cancer \\
\hline 9 & 79 & Female & No & 5.5 & Pleomorphic adenoma & Breast cancer \\
\hline 10 & 81 & Male & Yes & 4 & Not performed & Retroperitoneal lymphoma \\
\hline 11 & 63 & Male & Yes & 15.9 & Not performed & Lung cancer \\
\hline 12 & 65 & Male & Yes & 3.5 & Not performed & Oropharyngeal cancer \\
\hline 13 & 85 & Male & Yes & 13.1 and 9.9 & Not performed & Lung cancer \\
\hline 14 & 58 & Female & Yes & 6.8 & Not performed & Takayasu arteritis \\
\hline 15 & 65 & Male & Yes & 5.5 & Not performed & Lung cancer \\
\hline 16 & 70 & Male & Yes & 4.29 & Not performed & Lung cancer \\
\hline
\end{tabular}

Abbreviations: FNAC, fine needle aspiration cytology; PET/CT; positron emission/computed tomography; SUVmax, standardized uptake value.

Table 2 Data of parotidectomies performed in our department in the previous 10 years

\begin{tabular}{|l|l|l|}
\hline Histologic diagnosis & Number of cases (\%) & Smoking status (\%) \\
\hline Pleomorphic adenoma & $134(43.1 \%)$ & $52(38.8 \%)$ \\
\hline Warthin tumor & $80(25.7 \%)$ & $75(93.8 \%)$ \\
\hline Malignant tumors & $56(18 \%)$ & $31(55.4 \%)$ \\
\hline Other diagnosis & $41(13.2 \%)$ & $17(41.5 \%)$ \\
\hline Total & $311(100 \%)$ & $175(56.3 \%)$ \\
\hline
\end{tabular}

sample, Horiuchi et al detected four cases of abnormal parotid FDG uptake in 1,872 PET scans, with an estimated prevalence of $0.2 \%$; all four cases were diagnosed with WT. ${ }^{2}$ Other series of cases also found WT as the main cause of PGI. ${ }^{1,3}$

As PGIs are likely benign lesions, metastasic disease should not be suspected initially without histopathologic confirmation, even in patients with known malignancy elsewhere. ${ }^{1,4,5}$ Nevertheless, these lesions warrant further radiologic and histopathologic correlations. Ultrasonography and FNAC are useful tools to characterize parotid masses, avoiding erroneous staging of primary malignancies. The objective of cytologic diagnosis of parotid masses is the differential diagnosis between benign and malignant lesions. ${ }^{12}$ Complex histopathology and heterogeneity of cellular patterns of salivary gland tumors explain why typing of primary salivary malignancies by FNAC is a challenge. The accuracy of FNAC depends on the experience of the clinician who performs the procedure and the pathologist who evaluates cytologic material. ${ }^{12}$
We found the prevalence of smoking in parotid tumors surgically treated in our hospital was significantly higher in patients with WT than in patients with other tumors $(p<0.001)$. More than $90 \%$ of patients with WT were smokers, and cigarette use in patients with other benign and malignant neoplasms was lower, 38 and $55.4 \%$, respectively. Several other authors have studied the relation between WT and smoking. According to their publications, between 80 and $94 \%$ of patients with WT smoke, ${ }^{7-9,13}$ and the risk for development of WT correlates with the level and duration of smoking. ${ }^{7,9}$ Sadetzki et al found a remarkably high odds ratio (15.3) for developing WT in the presence of smoking. ${ }^{9}$

\section{Conclusion}

Based on the high prevalence of WT as PGI on PET/CT and the strong relationship with smoking, we suggest diagnosis other than Warthin tumor should be considered for PGI in 
nonsmokers. Further studies with histologic diagnosis and larger samples are warranted to confirm our hypothesis.

\section{References}

1 Lee SK, Rho BH, Won KS. Parotid incidentaloma identified by combined $18 \mathrm{~F}$-fluorodeoxyglucose whole-body positron emission tomography and computed tomography: findings at grayscale and power Doppler ultrasonography and ultrasound-guided fine-needle aspiration biopsy or core-needle biopsy. Eur Radiol 2009;19(9):2268-2274

2 Horiuchi M, Yasuda S, Shohtsu A, Ide M. Four cases of Warthin's tumor of the parotid gland detected with FDG PET. Ann Nucl Med 1998;12(1):47-50

3 Klijanienko J, Petras S, De Bosschere L, Paulmier B, Le Tourneau C, Rodriguez J. False-positive FDG PET/CT uptake in Warthin tumor in head and neck oncological patients confirmed by a fine needle aspiration. Diagn Cytopathol 2012;40(3):282-284

4 Enomoto A, Nakahara H, Uchihashi T, Tsuji H, Hamada S. Fluorodeoxyglucose-positive Warthin tumor in a neck node mimicking metastasis in primary intraosseous left posterior mandibular cancer staging with positron emission tomography/computed tomography. J Oral Maxillofac Surg 2011;69(7):2052-2054

5 Iwai T, Baba J, Shibasaki M, et al. 18F-fluorodeoxyglucose-positive Warthin tumor in a contralateral cervical lymph node mimicking metastasis in tongue cancer staging with PET/CT. J Craniofac Surg 2012;23(5):e507-e509

6 Coronado Poggio M, Couto Caro RM, Rodado Marina S, Martín Curto LM. 18F-FDG PET/TAC Semiology. Rev Esp Med Nucl 2008; 27(4):284-304, quiz 305-306

7 Peter Klussmann J, Wittekindt C, Florian Preuss S, Al Attab A, Schroeder U, Guntinas-Lichius O. High risk for bilateral Warthin tumor in heavy smokers-review of 185 cases. Acta Otolaryngol 2006;126(11):1213-1217

8 Pinkston JA, Cole P. Cigarette smoking and Warthin's tumor. Am J Epidemiol 1996;144(2):183-187

9 Sadetzki S, Oberman B, Mandelzweig L, et al. Smoking and risk of parotid gland tumors: a nationwide case-control study. Cancer 2008;112(9):1974-1982

10 Reddy VM, Thangarajah T, Castellanos-Arango F, Panarese A. Conservative management of Warthin tumour. J Otolaryngol Head Neck Surg 2008;37(5):744-749

11 Wang HC, Zuo CT, Hua FC, et al. Efficacy of conventional wholebody $18 \mathrm{~F}-\mathrm{FDG} \mathrm{PET} / \mathrm{CT}$ in the incidental findings of parotid masses. Ann Nucl Med 2010;24(8):571-577

12 Zbären P, Schär C, Hotz MA, Loosli H. Value of fine-needle aspiration cytology of parotid gland masses. Laryngoscope 2001;111(11 Pt 1):1989-1992

13 Chedid HM, Rapoport A, Aikawa KF, Menezes AdosS, Curioni OA. Warthin's tumor of the parotid gland: study of 70 cases. Rev Col Bras Cir 2011;38(2):90-94 